\title{
Bemerkung zu der Arbeit von Dr. G. Illing: Über einen eigenartigen Befund in den Glandulae vesiculares und den Glandulae ductus deferentis des Rindes. \\ Von
}

Dr. Heinrich Gerhartz.

In einer im letzten Heft dieses Archivs (Bd. 66, Heft 1, vom 22. April ds. J.) erschienenen Arbeit macht Illing Mitteilung über schon von $D$ is selhorst beobachtete, aber falsch gedeutete "eigentümliche, kugelige, bläschenförmige, grosse, durchsichtige, glasige Gebilde", die an der Basis des sekretorischen Epithels unter dem Niveau der Kernreihe liegen, und zwar auf der Membrana propria. Die Gebilde lösten sich bei der Behandlung mit Alkohol, Chloroform, Xylol und Farblösungen auf, sodass man dann die leere Zelle vor sich hatte. Härtung in Sublimat-Kochsalzlösung, Formalin und Zenkerscher Flüssigkeit konservierte sie, und in Osmiumsäuregemischen färbten sie sich intensiv schwarz. Illing schliesst daraus mit Recht, dass es sich um Fettzellen handelt, die sich durch eine besondere Anordnung, Form und Grösse und vor allem durch ihr Vorkommen von den gewöhnlichen Fettzellen unterscheiden.

Hierzu möchte ich bemerken, dass ich in einer erst jüngst in eben diesem Archive veröffentlichten Arbeit (65. Band, S. 666, „Anatomie und Physiologie der samenableitenden Wege der Batrachier") ebenfalls die durch die oben gegebenen Merkmale charakterisierten Körper gefunden und schon an dieser Stelle sie den Fettzellen zugewiesen habe. Diese Gebilde habe ich sowohl bei Rana als Triton beschrieben und sie mit der De- und Regeneration des sekretorischen Epithels in ursächlichen $\mathrm{Zu}-$ sammenhang gebracht.

Hier die betreffenden Belege; zunächst bezüglich $\mathrm{R}$ a $\mathrm{n} a$ :

S. 673. "Mazeriert man Gewebsstückchen, so sieht man helle, kugelige Gebilde am Zellenrande nach dem Lumen zu und runde feine Körner in den Zellen. Im frischen Präparate ist es nicht anders. Die kleinen Körner färben sich mit Hermannscher, Flemmingscher Lösung und in Osmiumsäuredämpfen tiefschwarz. Es ist nötig, sie in frisch angefertigten Präparaten zu untersuchen, da sie sich in Xylol-Damarlack bald auflösen und dann 
nur noch Lücken im Epithel zurückbleiben. Es liegt nahe, in den grösseren vor den Zellen liegenden Buckeln Sekretballen zu sehen und die anderen oben erwähnten kleinen Körnchen, die nicht zu allen Zeiten vorhanden sind, als Fettkörner anzusprechen." - Und ferner S. 681: „Im Epithel sieht man zahlreiche Fettkörnchen liegen, die auch schon im Februar sichtbar waren. Später sah ich sie nur noch einmal, Ende Juni, allerdings in sehr geringer Anzahl. Ob dies mit dem Hungerzustand in $\mathrm{Zu}-$ sammenhang $\mathrm{zu}$ bringen ist, vermag ich nicht $\mathrm{zu}$ entscheiden. In diesem Monat wurden nur Tiere untersucht, die längere Zeit gehungert hatten "

Bei Triton findet man analoge Befunde:

S. 689. „Eigentümlich sind tiefschwarze Körner, die die dunklen Zellen füllen - das Präparat war in Flemmingscher Lösung gehärtet - doch auch in den hellen nicht fehlen, aber dort nicht so intensiv tingiert sind. Ihre Gestalt wechselt von kleinsten rundlichen Körnern bis zu dicken Schollen mit unregelmåssigen Konturen. 'Im letzteren Falle sieht man sie meist in den dunklen Zylinderzellen liegen und hier oft den apikalen Zellenrand begrenzen. Sie können mitunter so an Zahl zunehmen, dass die ganze Struktur des Epithels von ihnen verdeckt wird. Auch in der umliegenden Muskulatur fehlen sie nicht; aber sie verlieren sich allmählich von der Epithelbasis, wo sie am dicksten sind, nach aussen hin. Sehr oft liegen sie hier in Reihen geordnet. Die Kerne bleiben frei von ihnen. Ich bin geneigt, diese Körner für Fett zu halten und in ihnen gleiche Elemente zu sehen, wie sie auch anderwärts eine Rolle spielen, wo es sich um die Fortschaffung von Organteilen handelt, welche, wie hier die exzessive Wucherung des Epithels, nur für eine liurze Spanne Zeit Bedeutung besitzen."

Meines Erachtens unterliegt es keinem Zweifel, dass die von Illing neu beschriebenen Fettzellen mit den oben erwähnten identisch sind, sodass es sich um eine weit verbreitete Einrichtung im sekretorischen Epithel der Samenwege zu handeln scheint 